\title{
Simplified shear relaxation modulus function of HDPE for FE simulation in extrusion blowing process
}

\author{
Nattarawee Siripath ${ }^{1, a}$ and Satjarthip Thusneyapan ${ }^{1}$ \\ ${ }^{1}$ Department of Mechanical Engineering, Faculty of Engineering, Kasetsart University, Bangkok, 10900, Thailand.
}

\begin{abstract}
Attempts to apply finite element analysis for simulation of the final geometry from extrusion blowing process have been studied. One of the accuracies for the prediction is a suitable mathematical model of the thermoplastic during the process. Visco-elastic model is found to be a suitable model by many researchers. This paper simplified the shear relaxation modulus function (SRMF) which characterizes the high-density polyethylene in molten state. Instead of the series of five exponential functions previously used, our simplified-SRMF consists of one power function of temperature and time. The deviation of our model from Williams-Landel-Ferry model with KBKZ constants, between the temperature of $140^{\circ} \mathrm{C}$ to $200^{\circ} \mathrm{C}$ and time from 0 to $10 \mathrm{sec}$, is $9.3706 \%$. When simulated on a bottle, our model has the final bottle thickness and time deviation from WLF-KBKZ model by $0.19622 \%$ and $3.07362 \%$ respectively.
\end{abstract}

\section{Introduction}

Research in employing finite element analysis (FEA) technology for simulation the extrusion blow molding process have been performed by many scholars [1-6]. The objective of the analysis is to find the wall thickness of products $[2,3,5,6]$. The thickness depends on the process conditions - mainly the parison conditions. The parison is a long hollow thermoplastic molten tube. Air pressure blows inside the parison; thus, expands the tube against the mold cavity surface to form the shape of products. The high-density polyethylene (HDPE) uses in extrusion blow molding process; its hot and soft parison exhibits visco-elasic characteristic. Hence, the isotropic visco-elastic model is good for modelling the deformation of HDPE parison in molten state $[5,6]$. The material property for the process simulation is shear relaxation modulus function or SRMF $[4,5,6]$. The time-temperature shift function introduced by WilliamsLandel-Ferry (WLF) is applied in SRMF [7]. The procedure of applying WLF together with KayeBernstein-Kearsley-Zapas (KBKZ) material constants introduces a series of five exponential functions $[4,5,6]$. This paper introduces a simplified SRMF as a function of temperature and time; it has only one power function.

\section{Mathematical model for HDPE}

Visco-elasticity is the property of HDPE, it represents both viscosity of fluid and elasticity of solid. In mechanical components, a viscous damper characterizes the viscosity, and an elastic spring is the elasticity. The mathematical model of the visco-elastic for finite element

\footnotetext{
${ }^{a}$ Corresponding author: s.natwee@gmail.com
}

(FE) simulation considers shear stress and shear strain [8]. A linear elastic material with shear elastic modulus of $G$, according to Hooke's law, shear stress of the elastic element $\tau_{e}$ relates to its shear strain $\gamma_{e}$ by

$$
\tau_{e}=G \gamma_{e} .
$$

The shear stress of the viscous material $\tau_{v}$ is characterrized by the shear strain rate $d \gamma_{v} / d t$ as

$$
\tau_{v}=\eta \frac{d \gamma_{v}}{d t},
$$

where $\eta$ is the viscosity and $\gamma_{v}$ is the shear strain in the damper (or viscous element).

Two basic visco-elastic models are Kelvin-Voigt and Maxwell. Since the deformation of the parison has more of viscous characteristic than elastic, the Maxwell material model is suitable for the application. The Maxwell model configuration has a damper in series with a spring. Under this circumstance, the total shear strain is

$$
\gamma=\gamma_{e}+\gamma_{v} .
$$

For a constant area, shear stress in the material $\tau$ equals to the shear stress of both elastic $\tau_{e}$ and viscous $\tau_{v}$ or

$$
\tau=\tau_{e}=\tau_{v} .
$$

The shear strain rate of the elastic element is found by differentiating Eq. (1), that is

$$
\frac{d \tau_{e}}{d t}=G \frac{d \gamma_{e}}{d t} .
$$

Since $\tau=\tau_{e}$ and from this equation, the shear strain rate of the elastic element is

$$
\frac{d \gamma_{e}}{d t}=\frac{1}{G} \cdot \frac{d \tau}{d t} .
$$


As $\tau=\tau_{v}$, and solving for the shear strain rate of Eq. (2), we have

$$
\frac{d \gamma_{v}}{d t}=\frac{\tau}{\eta}
$$

By taking the derivative of Eq. (3), we obtain

$$
\frac{d \gamma}{d t}=\frac{d \gamma_{e}}{d t}+\frac{d \gamma_{v}}{d t}
$$

Substituting Eqs. (5) and (6) into Eq. (7), the total shear strain rate becomes

$$
\frac{d \gamma}{d t}=\frac{1}{G} \cdot \frac{d \tau}{d t}+\frac{\tau}{\eta},
$$

which is a first-order differential equation of the form

$$
\dot{\tau}+\frac{G}{\eta} \tau=G \dot{\gamma}
$$

In the stress relaxation test, the strain is constant; then, strain rate $\dot{\gamma}$ is zero. Equation (8) becomes

$$
\dot{\tau}+\frac{G}{\eta} \tau=0
$$

The solution of the homogeneous differential equation of Eq. (9) is

$$
\tau(t)=\tau_{o} \exp \left(-\frac{t}{\Gamma}\right)
$$

where $\tau_{o}$ is the initial shear stress at time $t=0$, or $\tau_{o}=\tau(0)$, and

$$
\Gamma=\frac{\eta}{G} .
$$

The constant $\Gamma$ is the shear stress relaxation time; it is the ratio of the viscosity $\eta$ and the shear elastic modulus $G$ which has the unit of time. The shear stress relaxation time indicates the time taken the shear stress to relax or reaches zero; it is a dynamics property of the material.

Dividing Eq. (10) through by $\gamma$, we obtain the shear modulus as a function of time as

$$
G(t)=G_{o} \exp \left(-\frac{t}{\Gamma}\right) .
$$

For a material with several Maxwell models in parallel, each with viscosity $\eta_{i}$ and the shear stress relaxation time $\Gamma_{i}$, the shear stress relaxation modulus function (SRMF) is a Prony series as

$$
G(t)=\sum_{i=1}^{n} G_{i} \exp \left(-\frac{t}{\Gamma_{i}}\right) .
$$

For thermoplastic materials, the effect of temperature is described by time-temperature shift function $w(T)$ proposed by Williams-Landel-Ferry [4, 7]. The function $w(T)$ is known as WLF model, and is defined by

$$
w(T)=\exp \left(\frac{-c_{1}\left(T-T_{r e f}\right)}{c_{2}+\left(T-T_{r e f}\right)}\right) .
$$

The parameters of the function $w(T)$ are temperature coefficient $c_{1}$, temperature constant $c_{2}$ and the reference temperature $T_{r e f}$ in ${ }^{\circ} \mathrm{C}$. These constants for HDPE for the WLF function are in Table 1 [4]. Using the data in Table 1, time-temperature shift function $w(T)$ for HDPE is
Table 1. The HDPE constants of WLF function.

\begin{tabular}{|c|c|}
\hline Parameter & Value \\
\hline$c_{1}$ & 6.928 \\
\hline$c_{2}$ & $350^{\circ} \mathrm{C}$ \\
\hline$T_{r e f}$ & $150^{\circ} \mathrm{C}$ \\
\hline
\end{tabular}

$$
w(T)=\exp \left(\frac{-6.928(T-150)}{350+(T-150)}\right) \text {. }
$$

Notice that the high temperature $T$ reduces the value of the function $w(T)$. During the blowing process, the molten HDPE temperature is vary, and causes the change in the SRMF. This temperature affects the SRMF by introducing $w(T)$ as a multiplier of $\Gamma_{i}$ as shown in Eq. (16), and it becomes a function of two variables: temperature $T$ and time $t$. This time $t$ is referred to relaxation time.

$$
G(T, t)=\sum_{i=1}^{n} G_{i} \exp \left(-\frac{t}{\Gamma_{i} w(T)}\right) .
$$

The value of $\Gamma_{i}$ and $G_{i}$ of HDPE at $150^{\circ} \mathrm{C}$ according to the KBKZ material constants are shown in Table 2 [4].

Table 2. The KBKZ constants $\Gamma_{i}$ and $G_{i}$ of HDPE at $150^{\circ} \mathrm{C}$.

\begin{tabular}{|c|c|c|}
\hline$i$ & $\Gamma_{i}(\mathrm{sec})$ & $G_{i}(\mathrm{MPa})$ \\
\hline 1 & 0.03906 & 0.2475 \\
\hline 2 & 0.3125 & 0.07243 \\
\hline 3 & 2.5 & 0.03046 \\
\hline 4 & 20 & 0.007814 \\
\hline 5 & 160 & 0.003188 \\
\hline
\end{tabular}

The SRMF or $G(T, t)$ of HDPE of Eq. (16) by using data of $\Gamma_{i}$ and $G_{i}$ in Table 2 is

$$
\begin{aligned}
& G(T, t)=0.2457 \exp \left(-\frac{t}{0.03906 w(T)}\right) \\
& +0.07243 \exp \left(-\frac{t}{0.3125 w(T)}\right)+0.03046 \exp \left(-\frac{t}{2.5 w(T)}\right) \\
& +0.007814 \exp \left(-\frac{t}{20 w(T)}\right)+0.003188 \exp \left(-\frac{t}{160 w(T)}\right) .
\end{aligned}
$$

For the temperatures from $140^{\circ} \mathrm{C}$ to $200^{\circ} \mathrm{C}$ and relaxation times $t$ from 0.01 to 100 seconds, the values of the function $G(T, t)$ is plotted in Figure 1; some selected values are tabulated in Table 3 .

\section{Mathematical model of the simplified- SRMF}

The SRMF of Eq. (17) is a series of exponential functions and it is closed to be linear on log-log scale as shown in Figure 2a. With this characteristic, the SRMF can be expressed by a simple power function as

$$
G^{*}(T, t)=a t^{b},
$$

where $a$ is the coefficient of $t$, it is also a function of the temperature, or $a=a(T)$, and $b$ is the power of $t$, and is a function of the temperature, or $b=b(T)$. 

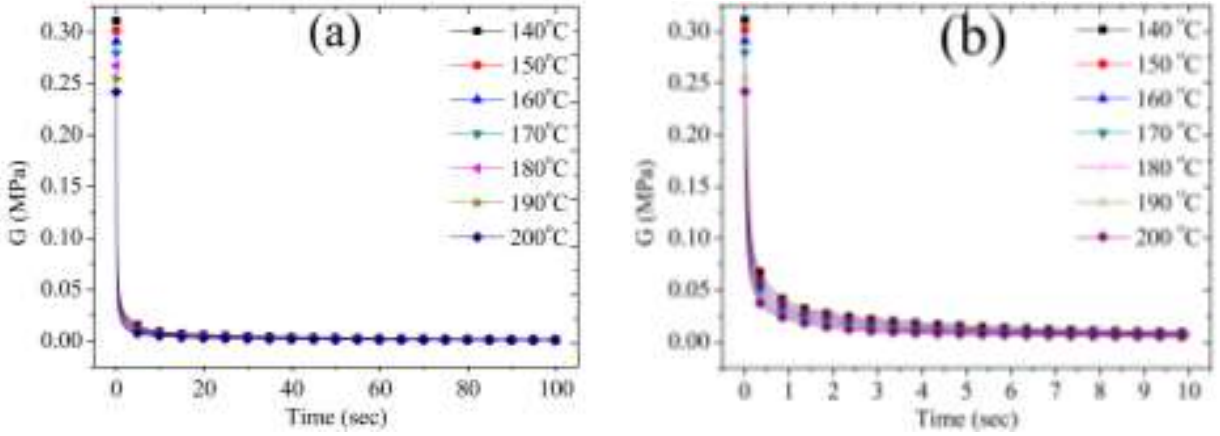

Figure 1. The shear relaxation modulus function $G(T, t)$ plots at the temperature from $140^{\circ} \mathrm{C}$ to $200^{\circ} \mathrm{C}$ and relaxation time from (a) 0 to 100 seconds, and (b) a close-up at 0 to 10 seconds.

Table 3. Tabulation of SRMF of Eq. (17), at five selected times $t$ from 0.01 to $100 \mathrm{sec}$, and the temperature from $140^{\circ} \mathrm{C}$ to $200^{\circ} \mathrm{C}$ with an increment of $10^{\circ} \mathrm{C}$.

\begin{tabular}{|c|c|c|c|c|c|}
\hline \multirow{2}{*}{$T\left({ }^{\circ} \mathrm{C}\right)$} & \multicolumn{5}{|c|}{$G(T, t), \mathrm{MPa}$} \\
\cline { 2 - 6 } & $\begin{array}{c}i=1 \\
\end{array}=0.01 \mathrm{sec}$ & $\begin{array}{c}i=2 \\
t=0.1 \mathrm{sec}\end{array}$ & $\begin{array}{c}i=3 \\
t=1 \mathrm{sec}\end{array}$ & $\begin{array}{c}i=4 \\
t=10 \mathrm{sec}\end{array}$ & $\begin{array}{c}i=5 \\
t=100 \mathrm{sec}\end{array}$ \\
\hline $140^{\circ} \mathrm{C}$ & 0.311318928 & 0.12668616 & 0.037979565 & 0.009392852 & 0.002047137 \\
\hline $150^{\circ} \mathrm{C}$ & 0.301688795 & 0.11181232 & 0.033971399 & 0.008292174 & 0.001759064 \\
\hline $160^{\circ} \mathrm{C}$ & 0.291129554 & 0.100143051 & 0.030771846 & 0.007456435 & 0.001512679 \\
\hline $170^{\circ} \mathrm{C}$ & 0.279737765 & 0.091096553 & 0.028140791 & 0.006778176 & 0.001290089 \\
\hline $180^{\circ} \mathrm{C}$ & 0.267641012 & 0.083967795 & 0.025868246 & 0.006185415 & 0.001084033 \\
\hline $190^{\circ} \mathrm{C}$ & 0.254993521 & 0.078103762 & 0.023808775 & 0.005640652 & 0.000893834 \\
\hline $200^{\circ} \mathrm{C}$ & 0.241969952 & 0.073007628 & 0.021884103 & 0.005130375 & 0.00072152 \\
\hline
\end{tabular}
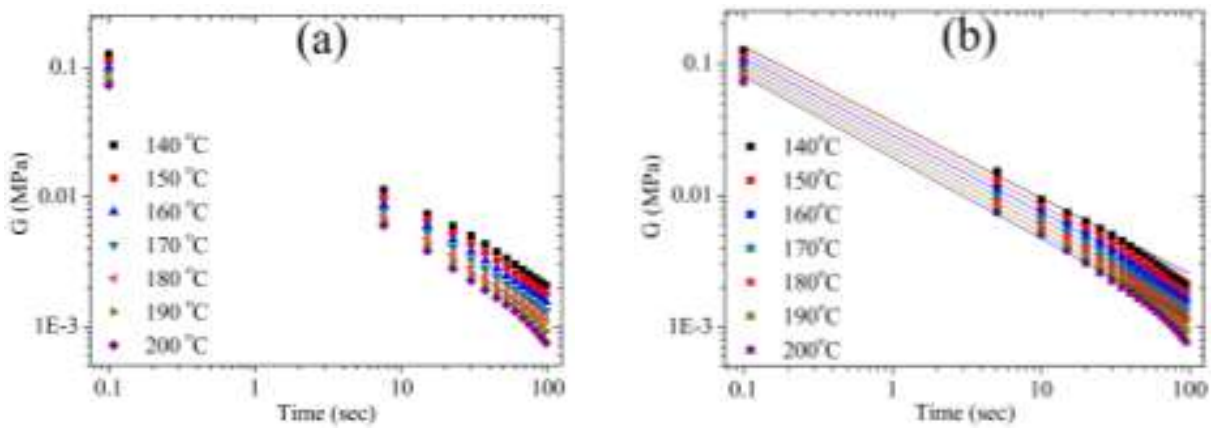

Figure 2. (a) Plot of $G(T, t)$ from Figure 1 on a log-log scale. (b) Comparing the linear least squares functions (21) to (27) with $G(T, t)$ at the 7 temperatures and the time to $100 \mathrm{sec}$.

Taking the natural logarithm on both sides of the equation, we have

$$
\begin{array}{cc} 
& \ln \left[G^{*}(T, t)\right]=\ln \left(a t^{b}\right), \\
\text { or } & \ln \left[G^{*}(T, t)\right]=\ln (a)+b \ln (t) .
\end{array}
$$

Eq. (19) is a linear function when we write it as

$$
G_{\ln }^{*}=A+b t_{\ln },
$$

where $G_{\ln }^{*}=\ln \left[G^{*}(T, t)\right], A=\ln (a)$ and $t_{\ln }=\ln (t)$.

By using the linear least squares fitting, we obtain the SRMF at each temperature from $140^{\circ} \mathrm{C}$ to $200^{\circ} \mathrm{C}$ as follows.

$$
\begin{aligned}
& G^{*}\left(140^{\circ} \mathrm{C}, t\right)=0.03619 t^{-0.57512}, \\
& G^{*}\left(150^{\circ} \mathrm{C}, t\right)=0.03223 t^{-0.58048}, \\
& G^{*}\left(160^{\circ} \mathrm{C}, t\right)=0.02890 t^{-0.58644}, \\
& G^{*}\left(170^{\circ} \mathrm{C}, t\right)=0.02607 t^{-0.59296}, \\
& G^{*}\left(180^{\circ} \mathrm{C}, t\right)=0.02363 t^{-0.59990}, \\
& G^{*}\left(190^{\circ} \mathrm{C}, t\right)=0.02151 t^{-0.60713}, \\
& G^{*}\left(200^{\circ} \mathrm{C}, t\right)=0.01963 t^{-0.61453},
\end{aligned}
$$

The graph of Eqs. (21) to (27) from time of 0 to 100 seconds with the superimposed data points from Figure $2 \mathrm{a}$ are shown in Figure $2 \mathrm{~b}$.

The coefficient $a$ of Eqs. (21) to (27) is plotted against the temperature as shown in Figure $3 \mathrm{a}$; between $140^{\circ} \mathrm{C}$ to $200^{\circ} \mathrm{C}$. The coefficient $a$ is shown to be linear with the temperature $T$. The variation of $a$ is approximated by a linear function

$$
a(T)=A_{a}+B_{a} T .
$$

By using the linear least squares method, it yields.

$$
a(T)=0.07858-0.000297214 T,
$$

For positive value of $G^{*}(T, t)$, the coefficient $a(T)$ must be positive; hence, the condition for the temperature is $\mathrm{T}<246.34^{\circ} \mathrm{C}$. The goodness of fitting, of Eq. (29) between $140^{\circ} \mathrm{C}$ and $200^{\circ} \mathrm{C}$, consists of the coefficient of multiple determination $\mathrm{R}^{2}$ and the standard error of the estimate $\sigma_{e}$; they are $\mathrm{R}^{2}=0.98181$ and $\sigma_{e}=0.005252$. The $\mathrm{R}^{2}$ value is almost one, and $\sigma_{e}$ is near zero, they 

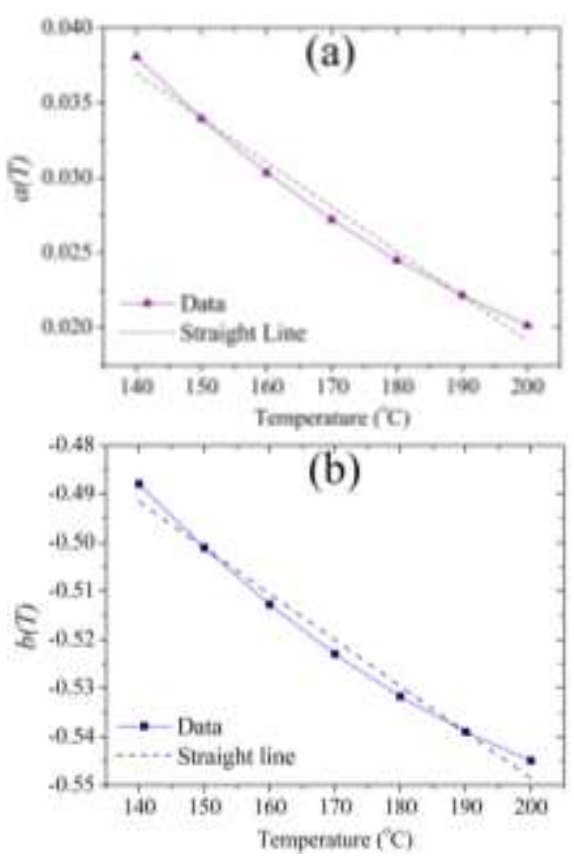

Figure 3. Plot of (a) the coefficient $a(T)$, and (b) the power $b(T)$. The straight lines are their linear approximation of functions (29) and (30) respectively.

indicate the good curve fitting of the linear function to the data.

Similarly, the power $b$ of Eqs. (21) to (27) is observed to be linear between $140^{\circ} \mathrm{C}$ and $200^{\circ} \mathrm{C}$, Figure $3 \mathrm{~b}$. The linear characteristic of $b$ is a function of the temperature $T$; by using the linear least squares calculation gives

$$
b(T)=-(0.35868+0.0009493574 T) .
$$

This function has $\mathrm{R}^{2}=0.97946$ and $\sigma_{e}=0.016777$. These two values indicate good fitting results.

Finally, the simplified-SRMF under the reference temperature at $150^{\circ} \mathrm{C}$ is.

$$
G^{*}(T, t)=(0.07858-0.00030 T) t^{-(0.35868+0.00095 T)} .
$$

Referring to Table 3, this function $G^{*}(T, t)$ is recommended for $140^{\circ} \mathrm{C} \leq T \leq 200^{\circ} \mathrm{C}$ and $0 \leq t \leq 100 \mathrm{sec}$.

The function $G^{*}(T, t)$ in Eq. (31) is our simplifiedSRMF (or S-SRMF) for the HDPE. The deviations of $G^{*}(T, t)$ from $G(T, t)$ vs. time of the 7 temperatures are shown in Figure 4a. The average of the 7 temperatures of the absolute deviation $\left|\Delta_{T} \%\right|$ at each time is shown in Figure $4 \mathrm{~b}$. The average deviations $\left|\Delta_{T} \%\right|$ against the temperature at $140^{\circ} \mathrm{C}$ to $200^{\circ} \mathrm{C}$ for the time from 0.01 to $10 \mathrm{sec}$ and 0.01 to $100 \mathrm{sec}$ are shown in Figure 4c; the overall averages are $9.3706 \%$ and $27.3335 \%$, respectively. Even though the deviation at $100 \mathrm{sec}$ is large, it has no great effect to the deviation; since most of the blow molding time is completed in less than 2 seconds.

\section{The use of S-SRMF for FEA thickness analysis}

In order to examine the accuracy of the S-SRMF in the FE simulation of the blowing process, a square bottle with the width, length and height of 76,76 and $170 \mathrm{~mm}$
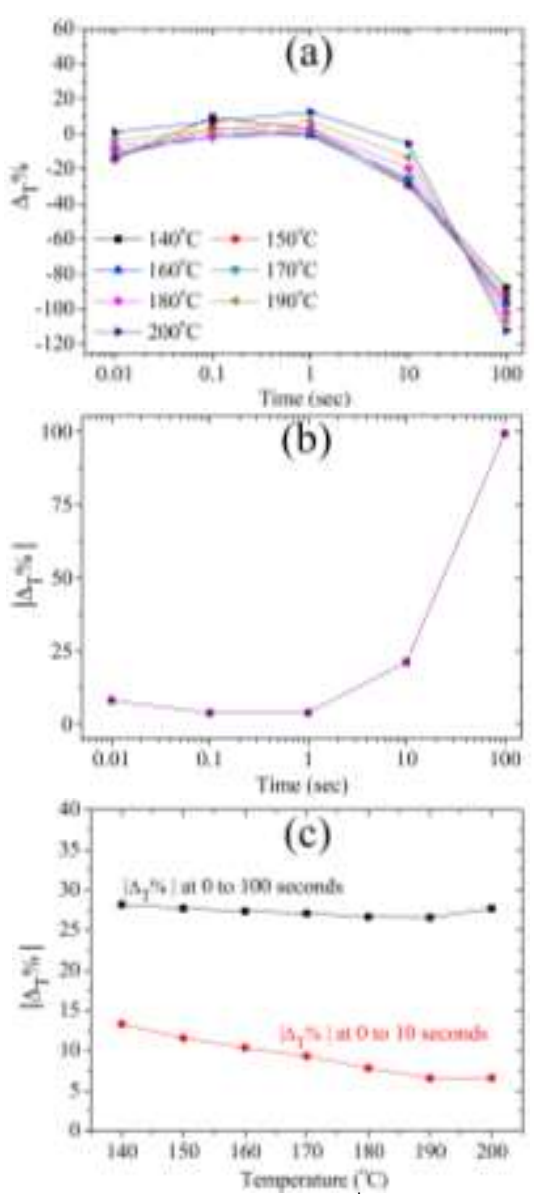

Figure 4. The percent deviations of $G^{*}(T, t)$ from $G(T, t)$. (a) $\Delta_{\mathrm{T}} \%$ vs. $T$ at $t=0.01$ to $100 \mathrm{sec}$. (b) Average of the absolute deviation $\left|\Delta_{T} \%\right|$ vs. $t$ of 7 temperatures. (c) Average of $\left|\Delta_{T} \%\right|$ vs. $T$ for time from 0.01 to $10 \mathrm{sec}$ and 0.01 to $100 \mathrm{sec}$.

was selected (Figure 5a). The FE model (FEM) for the parison, with the diameter of $38.5 \mathrm{~mm}$ and length of 185 mm, has 3504 nodes and 3456 elements (Figure 5b). Its quadrilateral element size is $2.5 \times 2.5 \mathrm{~mm}$ and $2.2 \mathrm{~mm}$ thick.

The isotropic visco-elastic model using WLF-KBKZ model $\left(G(T, t)\right.$ of Eq. (17)) and our S-SRMF, $G^{*}(T, t)$, of Eq. (31) were assigned as the material of the parison elements. The processing condition was set according to Rugsaj [6]. The FEA program for the simulation was MSC. MARC 2005. Figure $5 c$ is an illustration of the deforming parison at time $0.5,1.0,1.1,1.2$ and 1.5 seconds when using $G^{*}(T, t)$; note that the deform for $G(T$, $t$ ) has similar shape.

\section{Thickness deviation}

The comparison of the thickness was done at $0^{\circ}, 45^{\circ}$ and $90^{\circ}$ from the parting line (PL) and at $20 \%$ to $70 \%$ of the bottle height, as shown in Figure 6a. The graph in Figure $6 \mathrm{~b}$ compares the thickness, at the mentioned points, of $G^{*}(T, t)$ and $G(T, t)$. The thickness deviation between the two SRMFs in Figure $6 \mathrm{~b}$ has the maximum deviation of $0.85742 \%$. The average deviation along the $20 \%$ to $70 \%$ of the height at $0^{\circ}, 45^{\circ}$ and $90^{\circ}$ from PL are $0.05258 \%$, $0.38358 \%$ and $0.05005 \%$ respectively. 

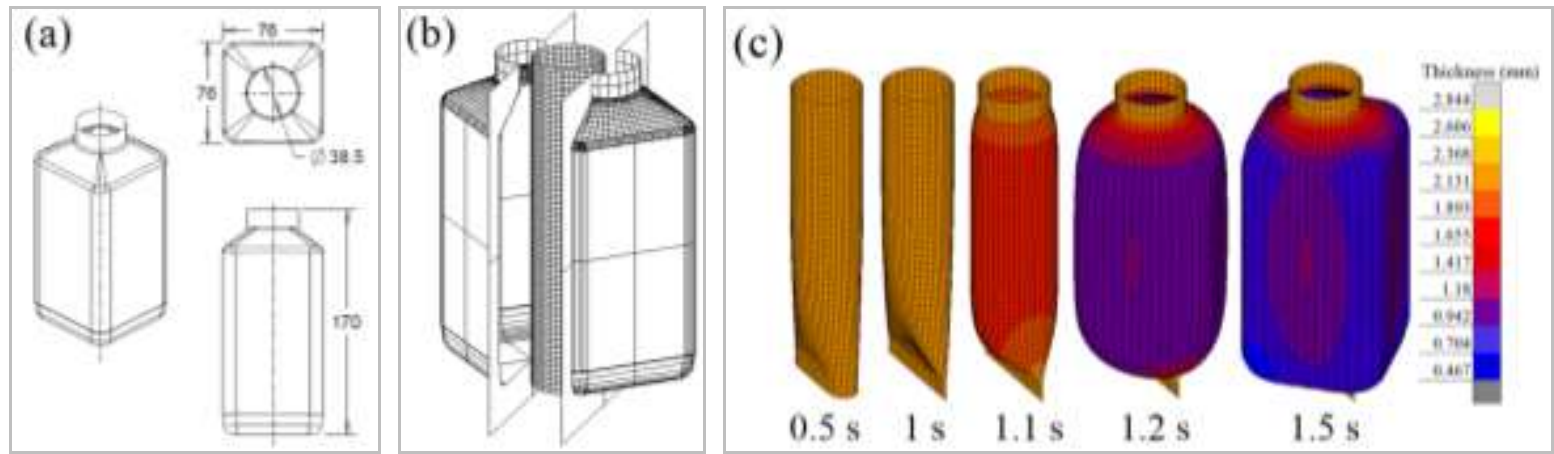

Figure 5. (a) Bottle dimension. (b) FEM of process. (c) Deforming parison at 0.5 to $1.5 \mathrm{sec}$.

(a)
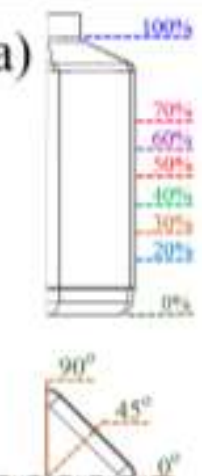
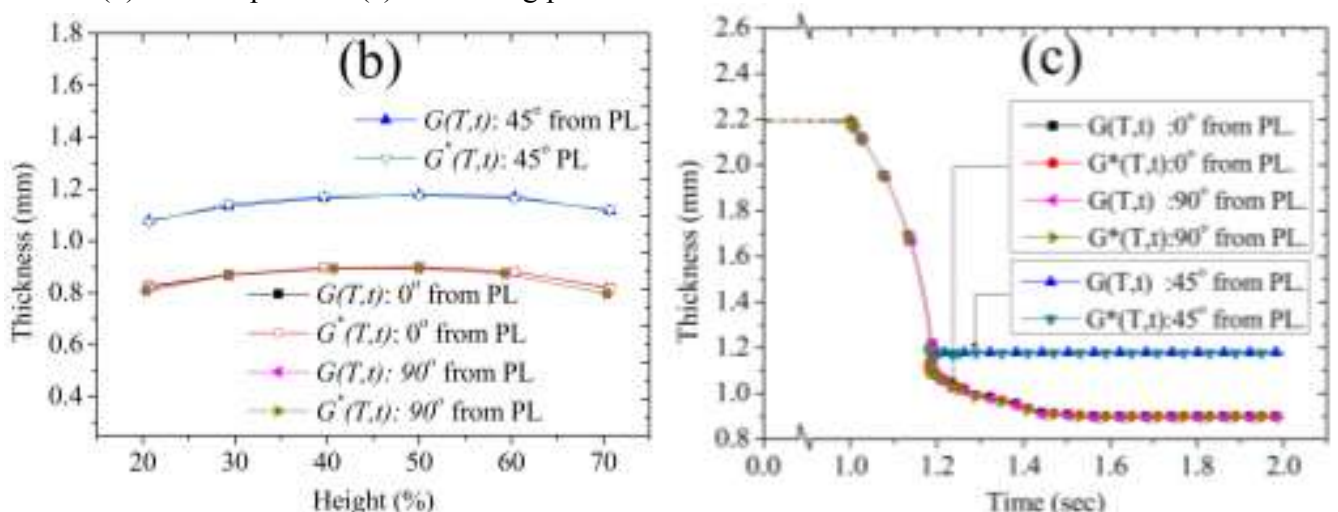

Figure 6. (a) The measuring points on the bottle. (b) Thickness obtained by using $G^{*}(T, t)$ and $G(T, t)$. (c) Thickness varying with time at $0^{\circ}, 45^{\circ}, 90^{\circ}$ and $50 \%$ height of $G^{*}(T, t)$ and $G^{*}(T, t)$.

The thickness varying with the blowing time at $0^{\circ}$, $45^{\circ}$ and $90^{\circ}$ from PL and $50 \%$ height is shown in Figure $6 \mathrm{c}$. The thickness responses of the model when using $G^{*}(T, t)$ and $G(T, t)$ look identical. The time deviations when the thicknesses become $1.2 \mathrm{~mm}$, at $0^{\circ}, 45^{\circ}$ and $90^{\circ}$ from PL are $0.03653,0.03653$ and $0.03653 \mathrm{msec}$, respectively; the average of this time difference is $0.03653 \mathrm{msec}$ or $3.073622 \%$.

The final thickness at $2 \mathrm{sec}$ of Figure $6 \mathrm{c}$ of the three points has the average deviation of $0.79686 \%$. The thicknesses are observed to begin to have constant thickness at time 1.2 and $1.5 \mathrm{sec}$. The results in these graphs indicated the compatibility of the S-SRMF with the model using WLF-KBKZ data.

\section{Conclusion}

The popularly used visco-elastic material model for extrusion blow molding for finite element simulation is based on WLF model and KBKZ data. Their shear relaxation modulus function or SRMF for HDPE contain a sum of five exponential functions of temperature and time. This paper presents a simplified-SRMF (S-SRMF) which has only one power function. The S-SRMF or $G^{*}(T, t)$ is numerically obtained from the data of WLF$\mathrm{KBKZ}$ from the temperature at $140^{\circ} \mathrm{C}$ to $200^{\circ} \mathrm{C}$, and time from 0 to $100 \mathrm{sec}$. Our S-SRMF is found easier for calculation and suitable for FE analysis. It has the average deviation from WLF-KBKZ data of $9.3706 \%$ for time from 0 to $10 \mathrm{sec}$. The FE simulation of a square bottle, our S-SRMF when compared with WLF-KBKZ model, has the final bottle thickness deviation of $0.19622 \%$ and the average time deviation when the thickness become $1.2 \mathrm{~mm}$ of $3.07362 \%$; therefore, the SSRMF gives a comparable result with the conventionally used SRMF for both thickness and time response.

\section{References}

1. S.I. Tanifuji, Process Poly. Eng. Sci., 40, 16 (2008)

2. K. Peplinski, Journal of Polish CIMAC, 5(3), 6 (2010)

3. K. Peplinski, Journal of Polish CIMAC, 7(3), 6, (2012)

4. D. Laroche, K.K. Kabanemi, L. Pecora, R.W. Diraddo, Poly. Eng. Sci. 39(7), 11 (1999)

5. S. Thusneyapan, R. Rugsaj, Journal of Research App. Mech. Eng., 4(1), 12-24 (2016)

6. R. Rugsaj, ME Thesis (Bangkok, Kasetsart University, 2014)

7. M.L. Williams, R.F. Landel, J.D. Ferry, J. Amer. Chem. Soc. 77, 7 (1955)

8. D.V. Rosato, A.V. Rosato, D.P. Dimattia, Blow Molding Handbook ( $2^{\text {nd }}$ revised edition $)$, Chapter 7 (2003) 